\title{
Alpha-synuclein fragments trigger distinct aggregation pathways
}

\author{
Tasnim Chakroun ${ }^{1,2}$, Valentin Evsyukov ${ }^{1,3}$, Niko-Petteri Nykänen ${ }^{1}$, Matthias Höllerhage $\mathbb{0}^{1,3}$, Andreas Schmidt ${ }^{4}$, \\ Frits Kamp ${ }^{5}$, Viktoria C. Ruf', Wolfgang Wurst ${ }^{3,7}$, Thomas W. Rösler ${ }^{1,2,3}$ and Günter U. Höglinger ${ }^{1,2,3,8}$
}

\begin{abstract}
Aggregation of alpha-synuclein (aSyn) is a crucial event underlying the pathophysiology of synucleinopathies. The existence of various intracellular and extracellular aSyn species, including cleaved aSyn, complicates the quest for an appropriate therapeutic target. Hence, to develop efficient disease-modifying strategies, it is fundamental to achieve a deeper understanding of the relevant spreading and toxic aSyn species. Here, we describe comparative and proof-ofprinciple approaches to determine the involvement of aSyn fragments in intercellular spreading. We demonstrate that two different aSyn fragments (1-95 and 61-140) fulfill the criteria of spreading species. They efficiently instigate formation of proteinase-K-resistant aggregates from cell-endogenous full-length aSyn, and drive it into different aggregation pathways. The resulting aggregates induce cellular toxicity. Strikingly, these aggregates are only detectable by specific antibodies. Our results suggest that aSyn fragments might be relevant not only for spreading, but also for aggregation-fate determination and differential strain formation.
\end{abstract}

\section{Introduction}

Synucleinopathies are a group of neurodegenerative diseases collectively characterized by intracellular protein inclusions containing alpha-synuclein $(\alpha \operatorname{Syn}) . \alpha$ Syn is a predominantly presynaptic, intrinsically unfolded protein of 140 amino acids (aa) ${ }^{1-3}$. Its primary structure comprises three distinct domains: (i) an $\mathrm{N}$-terminal domain (aa 1-60) that binds lipids and adopts alpha helical structures, (ii) a central domain known as non-amyloid component (NAC) (aa 61-95) involved in aggregation, and (iii) a C-terminal acidic tail (aa 96-140) accountable for most interactions with other proteins and small molecules ${ }^{4}$. Currently, the exact physiological functions of $\alpha$ Syn are still elusive. Nevertheless, it became clearer that

\footnotetext{
Correspondence: Günter U. Höglinger (Hoeglinger.Guenter@mh-hannover.de) 'Department of Translational Neurodegeneration, German Center for Neurodegenerative Diseases (DZNE), 81377 Munich, Germany

${ }^{2}$ Munich Cluster for Systems Neurology (SyNergy), University of Munich, 81377 Munich, Germany

Full list of author information is available at the end of the article

These authors contributed equally: Tasnim Chakroun, Valentin Evsyukov,

Thomas W. Rösler, Günter U. Höglinger

Edited by A. Verkharsky
}

$\alpha$ Syn has versatile functions, likely achieved by the distinct biophysical properties of its three domains ${ }^{5-9}$.

Intercellular transmission of $\alpha$ Syn was proposed as a mechanism for pathology propagation from one brain area to another ${ }^{10,11}$. Since aggregation of $\alpha$ Syn appears as a pivotal event in the pathogenesis of synucleinopathies, understanding the specific mechanisms that lead to accelerated aggregation is crucial. Interestingly, up to $15 \%$ of total $\alpha$ Syn in Lewy bodies (LBs) is truncated ${ }^{4,12}$. Moreover, truncation of $\alpha$ Syn seems to correlate with accelerated aggregation and pathology in cell and mouse models ${ }^{13-15}$. Remarkably, fragments are emerging as a potential common feature observed also for other neurodegeneration-related proteins, such as amyloid precursor protein and tau ${ }^{16}$.

We hypothesized that $\alpha$ Syn fragments might be implicated in intercellular spreading of pathology. Therefore, we focused on the four main events of the spreading process: (i) the release of $\alpha$ Syn species into the extracellular space, (ii) their uptake into naïve cells, (iii) their seeding capacity within the recipient cells and (iv) subsequent toxic effects. 
Lund human mesencephalic (LUHMES) cells were used throughout the study. They differentiate into mature and well-characterized human dopaminergic neurons, which makes them highly relevant for $\alpha$ Syn pathology ${ }^{17-19}$. Herein, we report that $\alpha$ Syn fragments are found in the extracellular space of $\alpha$ Syn-overexpressing cells. Furthermore, we describe two fragments that were rapidly taken up by cells and seem to mediate the aggregation of endogenous full-length $\alpha$ Syn (FL$\alpha$ Syn) into different pathways. The resulting aggregates were only detectable with specific antibodies, and ultimately induced toxicity.

\section{Results}

\section{aSyn fragments are present in conditioned medium of} aSyn-overexpressing neurons

To study the release of $\alpha$ Syn into the extracellular space, we used a previously reported $\alpha$ Syn overexpression model in differentiated LUHMES cells ${ }^{19}$. Cells were transduced with adenoviral (AV) vectors at day in vitro (DIV) 2 and toxicity, intracellular and extracellular $\alpha$ Syn were monitored over time (Fig. 1a). $\alpha$ Syn-mediated toxicity was assessed with lactate dehydrogenase (LDH) activity in culture medium (Fig. 1b). $\alpha$ Syn overexpression induced increased toxicity over time in comparison to GFPoverexpressing and untreated control cells, and reached approximately $50 \%$ at DIV8.

Next, we examined the intracellular $\alpha$ Syn species in whole cell homogenates (Fig. 1c). Expectedly, $\alpha$ Syn overexpression led to a stable increase in $\alpha$ Syn levels and marked intracellular aggregation. No intracellular $\alpha$ Syn fragments lower in molecular weight than FL- $\alpha$ Syn $(15 \mathrm{kDa})$ were detectable at any time after transduction.

We then analyzed cell conditioned medium (CM) for extracellular $\alpha$ Syn species (Fig. 1d). Several forms of $\alpha$ Syn appeared in the CM at DIV6; an oligomeric species at approximatively $37 \mathrm{kDa}$, monomeric $\mathrm{FL}-\alpha \mathrm{Syn}$ at $15 \mathrm{kDa}$ and several $\alpha$ Syn fragments ranging between 13 and $6 \mathrm{kDa}$. At DIV8, the level of all $\alpha$ Syn species increased considerably with concomitant increase of cytoplasmic leakage, pointing towards an accumulation of $\alpha$ Syn in the medium as a result of cell death.

\section{Multiple truncation events lead to extracellular aSyn fragments}

Next, we aimed to confirm that the $\alpha$ Syn-immunoreactive bands smaller than $15 \mathrm{kDa}$ in the extracellular space are indeed $\alpha$ Syn fragments, and to uncover their cleavage sites. Hence, we analyzed the CM of $\alpha$ Synoverexpressing cells at DIV8 by liquid chromatography tandem mass spectrometry (LC-MS/MS) (Fig. 2a). Peptides corresponding to $\alpha$ Syn were found in all investigated gel sections, except gel section \#7. One $\mathrm{N}$-terminal and multiple C-terminal truncation events were detected
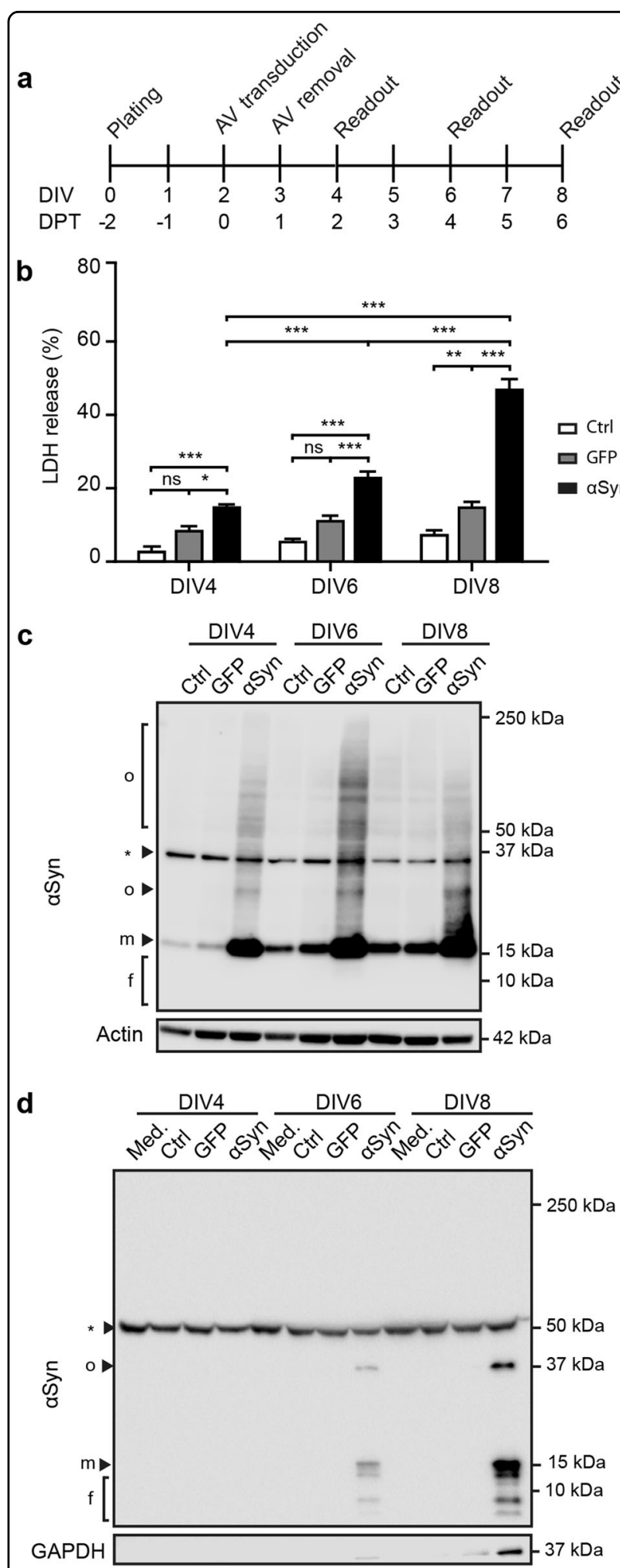

Fig. 1 (See legend on next page.) 
(see figure on previous page)

Fig. 1 aSyn fragments are present in the conditioned medium of aSyn-overexpressing LUHMES neurons. a Experimental design. LUHMES cells, grown in differentiation medium from plating onward, were transduced with GFP or aSyn adenoviral vectors (AV) two days after plating. Viruses were removed $24 \mathrm{~h}$ after transduction. Cells and conditioned medium (CM) were harvested at the indicated readout times. DIV: days in vitro. DPT: days post-transduction. $\mathbf{b}$ aSyn-mediated toxicity. Cellular toxicity was monitored by lactate dehydrogenase $(\mathrm{LDH})$ activity in the $\mathrm{CM}$ at the indicated readout times. Cells were either left untreated (Ctrl), challenged with a control AV expressing green fluorescent protein (GFP) or an AV expressing wild type aSyn. At DIV8, aSyn overexpression induced a considerably high toxicity, whilst GFP overexpression induced a mild toxicity, compared to untreated cells. LDH release values were related to a cell lysis positive control representing $100 \%$. Data are presented as mean + standard error of the mean (SEM) from at least 3 biological repeats. ns: not significant, ${ }^{*} p<0.05,{ }^{* *} p<0.005,{ }^{* * *} p<0.001$; two-way ANOVA with Tukey's post hoc test. c aSyn-overexpression in cell homogenates. Cell lysates were analysed by Western blot at the indicated readout times. aSyn overexpression levels were stable between DIV4 and DIV8.

Aggregation of aSyn is visible in overexpressing cells, but not in untreated control or GFP overexpression conditions. aSyn bands smaller than $15 \mathrm{kDa}$ were not detected, indicating absence of fragmented aSyn. f: fragments; m: monomer; o: oligomer; *: unspecific band. Actin was used as loading control. $\mathbf{d}$ aSyn in CM. Western blot analysis of aSyn in CM at the indicated times reveals the presence of several aSyn species at DIV6 and DIV8. An oligomer band appears at $37 \mathrm{kDa}(\mathrm{o})$, a monomer band at $15 \mathrm{kDa}(\mathrm{m})$ and several fragmented aSyn bands ranging from 13 to $6 \mathrm{kDa}(\mathrm{f})$. CM of untreated and GFP overexpressing cells did not contain detectable levels of aSyn. Unconditioned medium (Med.) was used as control for unspecific bands $\left({ }^{*}\right)$. GAPDH was used as control for cytoplasmic content in the $C M$.

(Fig. 2a). LC-MS/MS analysis confirmed the bands below $15 \mathrm{kDa}$ observed in CM (Fig. 1d) as several $\alpha$ Syn fragments (Fig. 2a), and suggest that they result from proteolytic activity at multiple sites of $\alpha$ Syn. However, it was not possible to identify precise cleavage sites which would have allowed to delineate the protease(s) responsible for this cleavage pattern in our model.

\section{aSyn fragments are generated extracellularly and further taken up by naïve cells}

Since $\alpha$ Syn fragments were observed exclusively in the extracellular space (Fig. 1c, d), we investigated whether they are generated extracellularly. Incubation of recombinant FL- $\alpha$ Syn with unconcentrated conditioned medium, followed by WB analysis with $\mathrm{N}$ - and C-terminal $\alpha$ Syn antibodies revealed that cleavage of recombinant $\alpha$ Syn occurred in CM of both $\alpha$ Syn-overexpressing and untransduced control cells, whereas the medium alone and the tested supplements did not lead to the cleavage of $\alpha$ Syn. In this experimental design, CM-derived FL- $\alpha$ Syn and fragments were below the detection limit. Therefore, only added recombinant FL- $\alpha$ Syn and its cleavage products could be visualized (Fig. 2b). The presence of cleaved $\alpha$ Syn in untransduced controls indicates that $\alpha$ Syn is very likely cleaved in the extracellular space by a protease that is secreted by cells under physiological conditions.

We next asked whether the fragments generated in the extracellular space of $\alpha$ Syn-overexpressing cells are taken up by naïve cells. To eliminate endogenous $\alpha$ Syn signal, we used $\alpha$ Syn knockout (KO) LUHMES neurons as recipient cells (Fig. 2c). An $\alpha$ Syn KO LUHMES cell line was generated by CRISPR/Cas9 genome editing and showed absence of $\alpha$ Syn throughout differentiation (Supplementary Fig. S1). For uptake evaluation, naïve $\alpha$ Syn KO cells were incubated with several concentrations of $\mathrm{CM}$ of wild-type (WT) aSyn-overexpressing cells. A dosedependent uptake of monomeric FL- $\alpha$ Syn and a $37 \mathrm{kDa}$ oligomer was observed, but no fragments could be detected upon short exposure time (Fig. 2c). With a longer exposure time, however, a signal corresponding to an uptake of $\alpha$ Syn fragments was visible with the highest concentrations of $\mathrm{CM}$ (5X and 10X). This finding provides evidence that $\alpha$ Syn fragments can be taken-up by naïve cells. Since in these experimental conditions the uptake of FL and oligomeric $\alpha$ Syn species prevailed over fragmented $\alpha$ Syn, we conducted further experiments with recombinant $\alpha$ Syn fragments.

\section{aSyn fragments differentially influence the aggregation of full-length aSyn}

To characterize the effects of $\alpha$ Syn fragments on FL$\alpha$ Syn aggregation by a systematic approach, distinct recombinant fragments containing one or two adjacent full domains of $\alpha$ Syn were chosen (Fig. 3a). Noteworthy, the fragments identified in sections \#3 and \#6 in the CM of $\alpha$ Syn-overexpressing cells (Fig. $2 \mathrm{a}$ ) were very similar to recombinant fragments 1-95 and 1-60.

In a cell-free aggregation assay, non-aggregated recombinant fragments were used as seeds and monomeric FL- $\alpha$ Syn was used as a substrate with a molar ratio of seed to substrate of 1 to 10 in all the reactions. $\alpha$ Syn preformed fibrils (PFFs) represent a positive control for seeded aggregation due to their well-established capacity as highly efficient seeds ${ }^{20,21}$ and FL- $\alpha$ Syn represents the baseline reference for aggregation kinetics.

The incubation of seeds without substrate showed that they do not have a considerable input on the aggregation curves (Fig. 3b). When the substrate was added to the reactions, the ThT signal increased substantially indicating that it corresponds primarily to the aggregation of FL- $\alpha$ Syn (Fig. 3c). Generally, the obtained aggregation curves had a characteristic sigmoidal amyloid aggregation shape (Fig. 3c). The apparent lag time was reported as the time at which ThT signal reached $10 \%$ of its total increase, and growth rates were calculated as the 


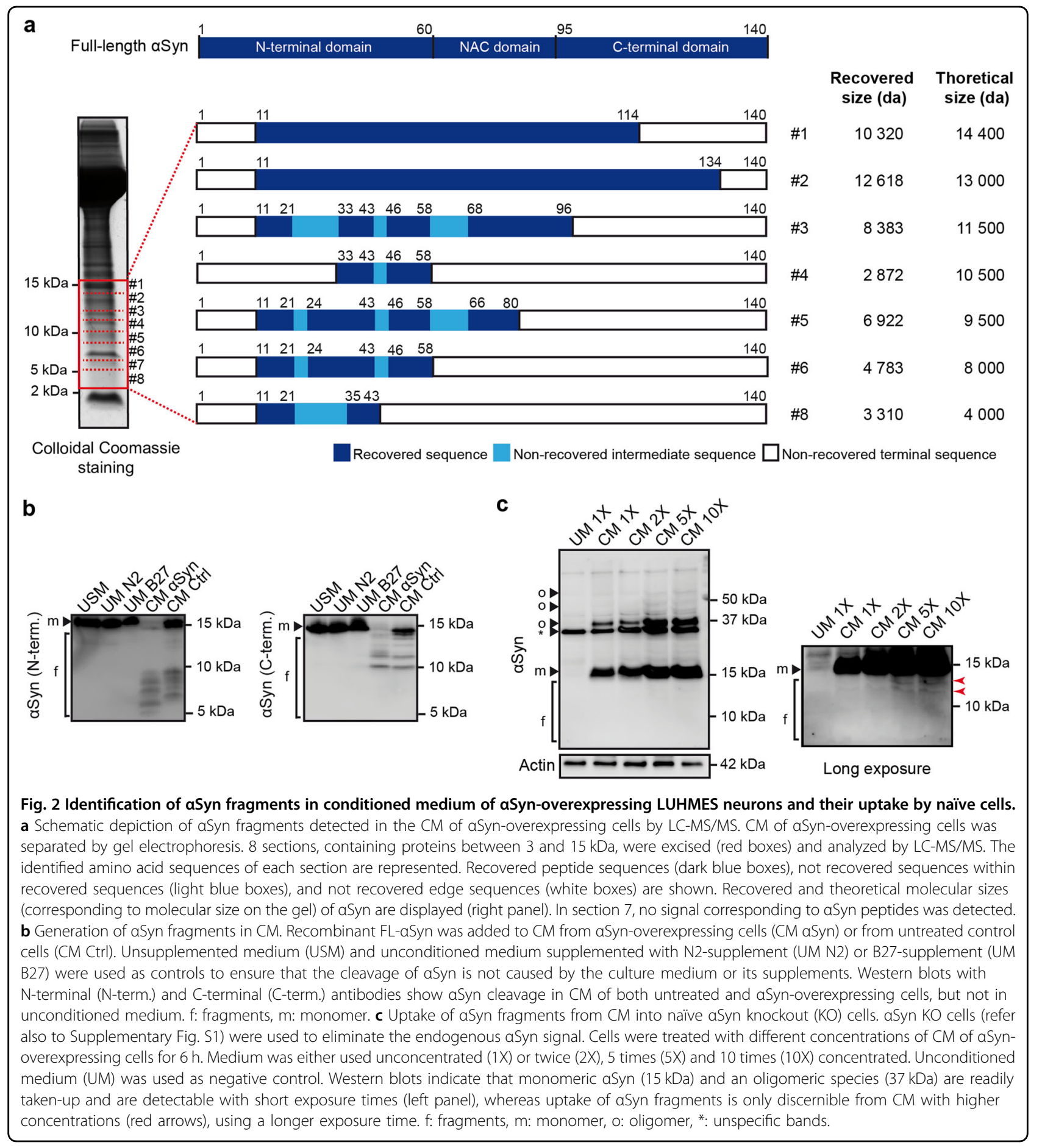

difference in RFU per hour between 10 and $50 \%$ of the total ThT signal increase (Fig. 3d).

As expected, PFFs dramatically accelerated the aggregation. Strikingly, fragment 1-95 behaved similarly and reduced the apparent lag time to the same extent as PFFs. Fragments 61-140 and 61-95 also accelerated the aggregation but more moderately. The growth rate of seeded aggregation with PFFs was higher than the one seeded with fragment 1-95 implying that even though both initiated aggregation with a very similar efficiency, the aggregation seeded with fragment 1-95 required a longer time to reach a plateau as compared to PFFs. Similarly, fragments 61-140 and 61-95 reduced the lag time without detectable effect on the growth rate in comparison with monomeric FL- $\alpha$ Syn. Fragments 1-60 and 96-140 had no detectable effect on aggregation kinetics (Fig. 3e, f). 


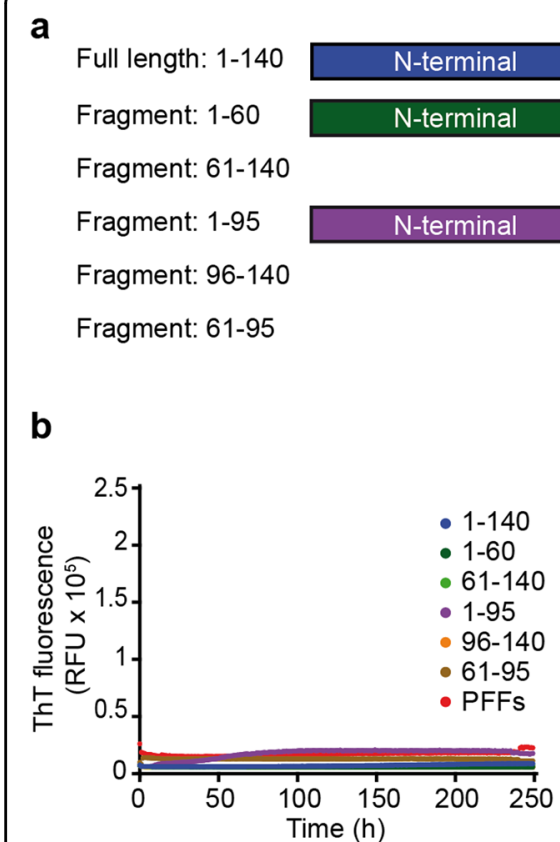

C

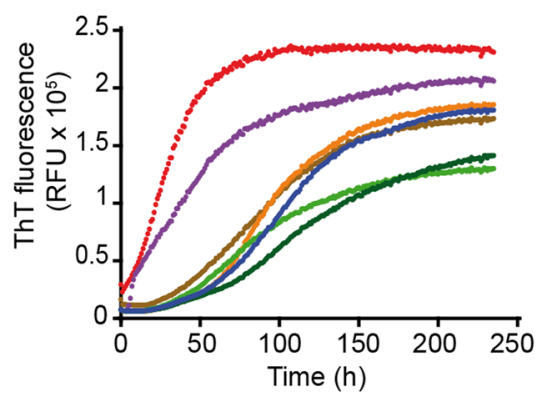

d

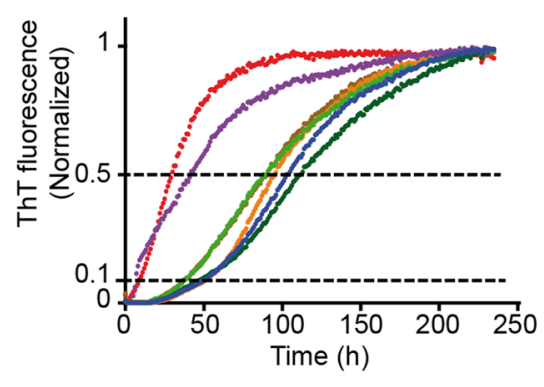

e

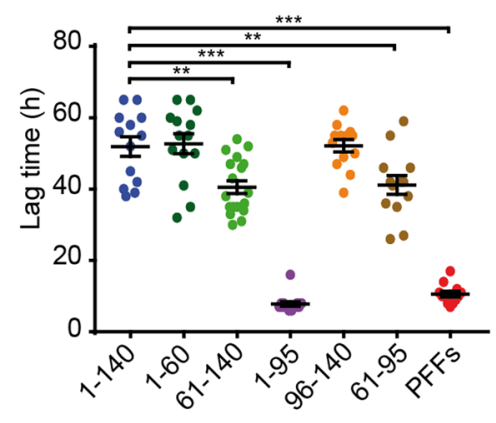

f

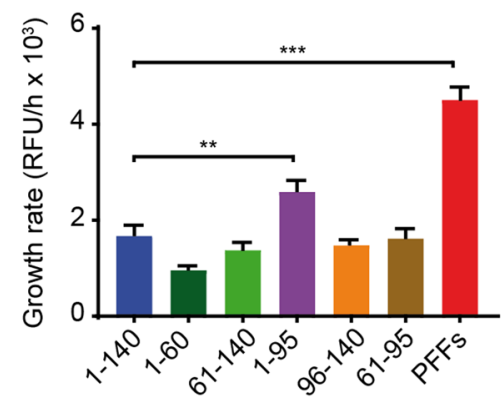

g

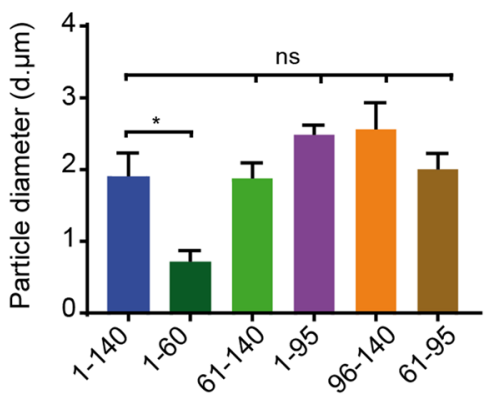

h

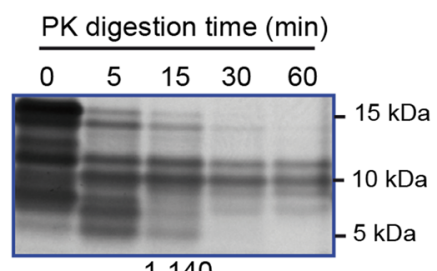

\section{NAC}

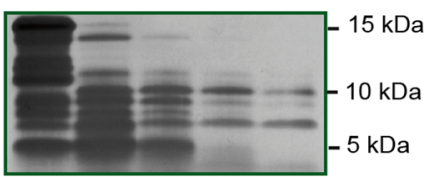

$1-140+1-60$

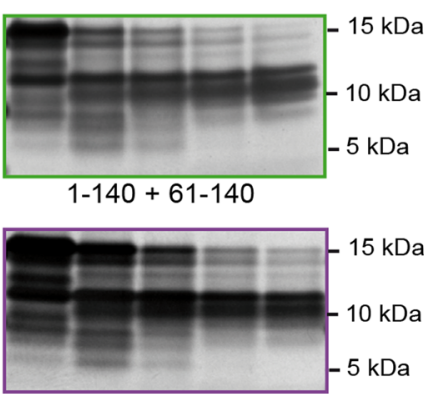

$1-140+1-95$

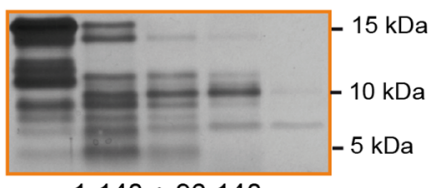

$1-140+96-140$

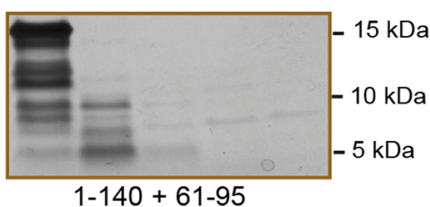

i

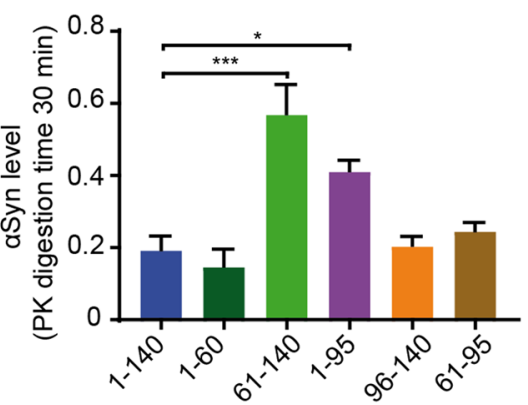

Fig. 3 (See legend on next page.)

Next, we studied whether seeding with different $\alpha$ Syn fragments would result in aggregates with different properties. Dynamic light scattering was used to measure the particle diameter of the different aggregates. No significant differences in particle size between the different aggregates were observed, except for the ones seeded with 
(see figure on previous page)

Fig. 3 Recombinant aSyn fragments show differential effects on aggregation of full-length aSyn in a cell-free aggregation assay.

a Schematic overview of recombinant FL-aSyn and fragments used in the study: Full length (1-140), N-terminal fragment (1-60), NAC + C-terminal fragment (61-140), N-terminal + NAC fragment (1-95), C-terminal fragment (96-140) and NAC fragment (61-95). The same names and color-code are used in all the panels. b-d Seeding effect of recombinant aSyn fragments on recombinant FL-aSyn in a cell-free aggregation assay. For the aggregation reactions, different fragments described in a were used as seeds and FL-aSyn was used as a substrate at a molar ratio of 1:10, respectively. Aggregation kinetics was monitored by measurement of thioflavin T (ThT) signal. aSyn pre-formed fibrils (PFFs) were used as positive control for seeding. RFU: relative fluorescence unit. Aggregation curves of aSyn fragments without addition of FL-aSyn substrate are shown in b. Aggregation curves with addition of FL-aSyn substrate as raw fluorescence data are shown in c, and normalized fluorescence ratio data from $\mathbf{c}$ are shown in $\mathbf{d}$. The lower $(0.1)$ and the upper (0.5) fluorescence thresholds of the total signal are shown with dotted lines. Aggregation curves are presented as mean from at least 3 repeats with 3 technical replicates each. e Lag time of aggregation seeded with different aSyn fragments. Lag times are presented as the time at which each curve reached $10 \%$ of the total fluorescence signal. Lag times were strikingly reduced in aggregation reactions seeded with PFFs and fragment 1-95. Each dot of the scatter plot represents one aggregation curve. Data are presented as mean \pm SEM from at least 3 repeats with 3 technical replicates each (black lines). ${ }^{* *} p<0.005$, ${ }^{* * *} p<0.001$; one-way ANOVA with Tukey's post hoc test. f Growth rate of aggregation seeded with different aSyn fragments. Aggregation growth rate is presented as a measure of increased ThT fluorescence per hour. Growth rates were significantly higher in aggregation seeded with the fragment 1-95 and the PFFs (positive control), and remained unchanged in all other aggregation reactions. Data are presented as mean + SEM from at least 3 repeats with 3 technical replicates each. ${ }^{* *} p<0.005,{ }^{* * *} p<$ 0.001; one-way ANOVA with Tukey's post hoc test. $\mathbf{g}$ Particle size of aggregates seeded with different aSyn fragments. Particle size of aggregates was measured with dynamic light scattering (DLS) after the aggregation reactions were finished. Except for fragment 1-60, which resulted in significantly smaller fibrils, no significant differences in fibril size were observed across the rest of the samples. Data are presented as mean + SEM of particle diameter (d.nm) from at least 3 repeats. ${ }^{*} p<0.05$; one-way ANOVA with Tukey's post hoc test. $\mathbf{h}$, i Proteinase K (PK) resistance of aggregates produced by seeded aggregation with different aSyn fragments. Aggregation was carried out with the same molar ratio of seed to substrate (1:10) as described above. PK digestion was performed at $37^{\circ} \mathrm{C}$ for the indicated amounts of time. Protein separation by gel electrophoresis followed by silver staining revealed that aggregates seeded with fragments 61-140 and 1-95 were more PK resistant than the other samples. i A normalized optical density quantification of aSyn digestion at $30 \mathrm{~min}$. Data are presented as mean + SEM from at least 3 repeats. ${ }^{*} p<0.05$, ${ }^{* * *} p<0.001$; one-way ANOVA with Tukey's post hoc test.

fragment 1-60 (Fig. 3g). Interestingly, both fragments 61-140 and 1-95 resulted in aggregates with a higher PK resistance (Fig. 3h, i). PFFs were not used as seeds in these latter experiments, since the aggregates formed by FL$\alpha$ Syn are identical to PFFs.

Taken together, these data showed that the NAC domain is essential for aggregation, and non-aggregated $\alpha$ Syn fragments that contain the NAC domain flanked by either the $\mathrm{N}$ - or the $\mathrm{C}$-terminal domain can act as effective seeds that differentially influence the aggregation of FL- $\alpha$ Syn.

\section{aSyn fragments are taken up by LUHMES neurons}

To investigate the relevance of recombinant $\alpha$ Syn fragments in intercellular spreading, we addressed their uptake into human dopaminergic neurons. Recombinant FL- $\alpha$ Syn and fragments were fluorescently labeled with ATTO-488 and successful labeling was verified with gel electrophoretic separation followed by fluorescence imaging (Fig. 4a).

Cells were treated at DIV4 for the indicated amounts of time and intracellular fluorescence was measured (Fig. 4b). Uptake was rapid during the first $12 \mathrm{~h}$, followed by a slower phase between 12 and $48 \mathrm{~h}$. The uptake kinetics of all labeled species was similar except for fragment 61-95, i.e. the NAC domain, which appears to be taken up less efficiently most likely because of its highly hydrophobic nature.
Uptake at $48 \mathrm{~h}$ after treatment was further ascertained with confocal imaging (Fig. 4c). The fluorescent signal corresponding to the different labeled $\alpha$ Syn species was contained within the cells, thus showing their intracellular localization.

\section{Fragments 1-95 and 61-140 induce different aggregation patterns}

The relevance of $\alpha$ Syn species in spreading depends on their capacity to seed intracellular aggregation after uptake. Therefore, we investigated whether the recombinant $\alpha$ Syn fragments have seeding potential on cellendogenous FL- $\alpha$ Syn. Since the physiological neuronal concentration of $\alpha$ Syn was previously estimated to be at least $30 \mu \mathrm{M}^{22,23}$, we treated differentiated LUHMES neurons with $3 \mu \mathrm{M}$ recombinant FL- $\alpha$ Syn and fragments to maintain the 1:10 seed to substrate ratio. Intracellular $\alpha$ Syn was examined using antibodies targeting the $\mathrm{N}$ - or $\mathrm{C}$-terminus of $\alpha$ Syn. We chose two readout times to analyze the immediate effects (DIV6) and the persistent effects after removal of treatments (DIV10) (Fig. 5a).

At DIV6, intracellular aggregation was only observed in cells exposed to FL- $\alpha$ Syn and fragments $61-140$ and 1-95 (Fig. 5b). Interestingly, the treatments resulted in different aggregation patterns, implying different aggregation pathways. Also, fragment 1-60 seemed to trigger a less prominent, distinct aggregation pathway. Fragments 96-140 and 61-95 did not induce detectable intracellular 


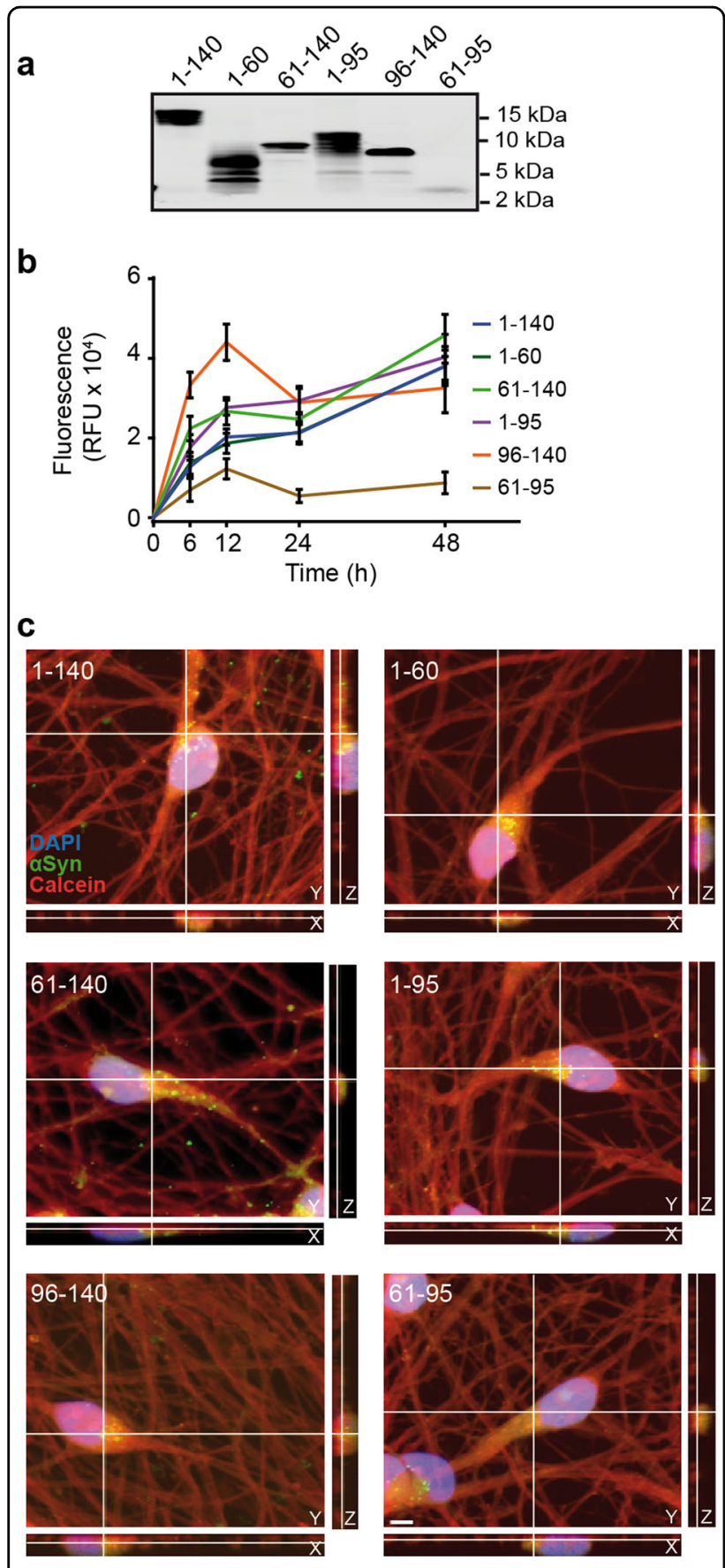

Fig. 4 Recombinant aSyn fragments are readily taken-up by naïve LUHMES neurons. a Fluorescent gel imaging of recombinant ATTO-488-labeled FL-aSyn and fragments (depicted in Fig. 3a). All labeled aSyn species have a fluorescent signal at the expected molecular size. $\mathbf{b}$ Uptake kinetics of recombinant FL-aSyn and fragments. Cells were treated with labeled aSyn at DIV4. At the indicated times, intracellular fluorescence was measured. Extracellular fluorescence was quenched using trypan blue. RFU: relative fluorescence unit. c Uptake of ATTO-488-labeled aSyn fragments $48 \mathrm{~h}$ after treatment. Confocal images show orthogonal projections of Z-stacks with a clear intracellular localization of recombinant FL-aSyn and fragments. A calcein red-orange filling (red), DAPI staining (blue) and labeled recombinant aSyn (green) are shown. Scale bar: $5 \mu \mathrm{m}$. aggregation, which may be due to the lack of the aggregation-prone NAC domain in the former, and a highly insoluble state and inefficient uptake for the latter (Fig. 4b, c).

Importantly, while intracellular aggregation ensuing from FL- $\alpha$ Syn treatment was detected by both $\mathrm{N}$ - and $\mathrm{C}$-terminal antibodies, aggregates resulting from treatment with fragments 1-95 and 61-140 were only detectable by the $\mathrm{N}$-terminal or the $\mathrm{C}$-terminal antibody, respectively. This intriguing finding could indicate that endogenous $\alpha$ Syn is recruited for aggregation, but the generated aggregates only expose seed-specific epitopes. Alternatively, intracellular aggregation observed at DIV6 potentially resulted from self-aggregation of the different $\alpha$ Syn treatments.

\section{Endogenous aSyn is required for intracellular fragment- seeded aggregation}

To clarify whether endogenous $\alpha$ Syn is recruited for aggregation seeded by fragments $61-140$ and $1-95$, we compared the intracellular seeding activity in WT and $\alpha$ Syn KO cells. At DIV6, the characteristic aggregation patterns were present in WT cells, whereas they either completely failed to appear for FL- $\alpha$ Syn and fragment 1-95, or were substantially reduced for fragment $61-140$ in $\alpha$ Syn KO cells (Fig. 5c). This indicates that endogenous $\alpha$ Syn is not merely recruited for fragment-seeded aggregation, but is essential for it to occur. Notably, the presence or absence of endogenous $\alpha$ Syn seems to influence either the uptake or the intracellular processing of recombinant $\alpha$ Syn fragments. In WT cells, FL- $\alpha$ Syn and fragments $61-140$ and 1-95 were clearly visible at the expected molecular size with short exposure times. In $\alpha$ Syn KO cells, longer exposure times had to be applied in order to visualize them (Supplementary Fig. S2).

Interestingly, aggregation seeded with FL- $\alpha$ Syn and fragments 61-140 and 1-95 continued for at least four days after treatment removal in WT cells (Fig. 5c). This emphasizes that endogenous $\alpha$ Syn is seemingly the fundamental substrate for maintaining the aggregation process. Moreover, these aggregates exhibited a clear shift of $\alpha$ Syn from soluble to insoluble state between DIV6 and DIV10 (Supplementary Fig. S3).

Furthermore, in accordance with our findings under cell-free conditions (Fig. 3h), intracellular aggregates seeded by fragments $61-140$ and 1-95 showed greater PK resistance than aggregates seeded with FL- $\alpha$ Syn (Fig. $5 d$ ).

\section{Fragments $61-140$ and 1-95 induce toxicity}

To investigate the relevance of $\alpha$ Syn fragments for pathology, we examined whether these specific fragments induced toxicity subsequent to intracellular aggregation. Therefore, we monitored the cellular toxicity by measuring LDH activity in the CM over time (Fig. 6a). None 


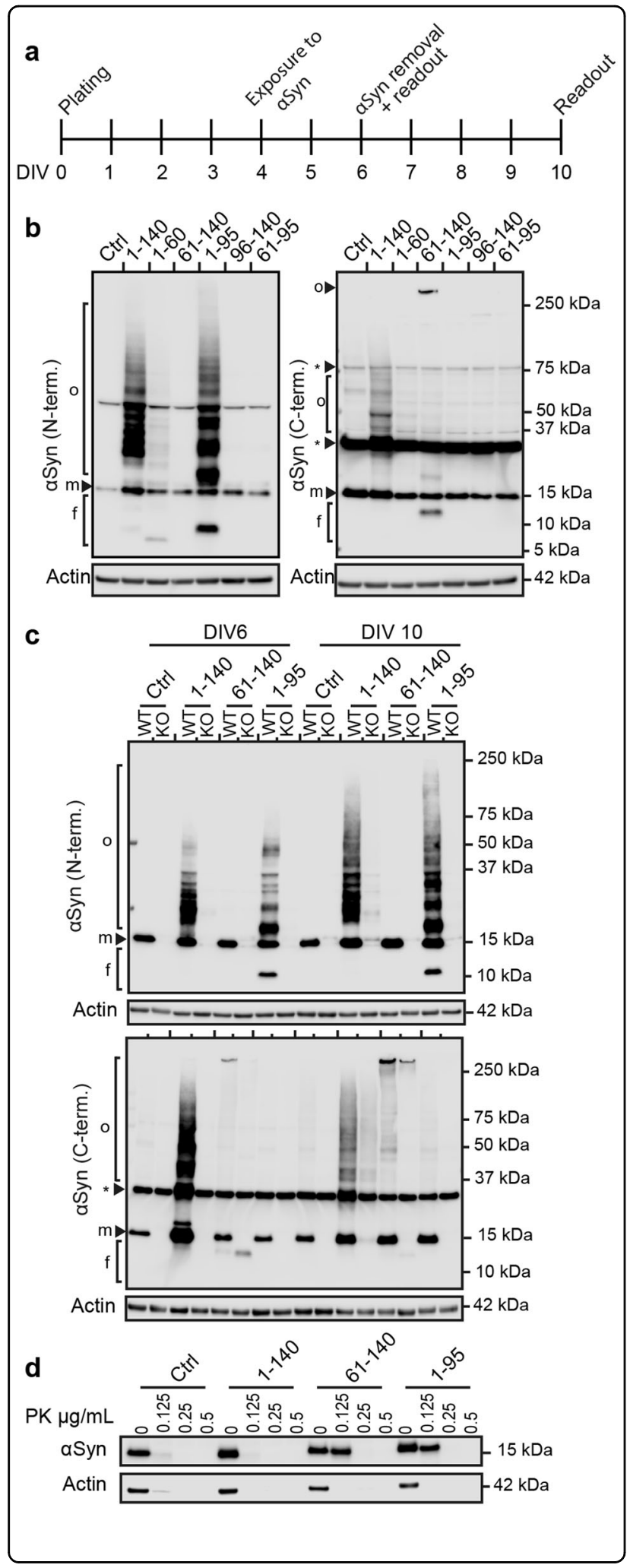

of the treatments showed an immediate toxic effect at DIV6 and DIV9 (Fig. 6b, c). However, only fragments 61-140 and 1-95 induced toxicity that first appeared at
Fig. 5 aSyn fragments induce different aggregation patterns in LUHMES neurons. a Experimental design. Cells were differentiated for 4 days before treatment. Treatments with recombinant FL-aSyn and fragments were carried out for $48 \mathrm{~h}$ and then removed. Two readout time points DIV6 and DIV10, were chosen. $\mathbf{b}$ Intracellular seeding potency of recombinant FL-aSyn and fragments at DIV6. Western blot analysis was performed with antibodies targeting either the $\mathrm{N}$-terminal domain $(\mathrm{N}$-tem.) or the $\mathrm{C}$-terminal domain (C-term.) of aSyn. Fragment 1-95 induced intracellular aggregation which could only be visualized by an N-terminal aSyn antibody, whereas fragment 61-140 induced intracellular aggregation which only could be visualized by a C-terminal aSyn antibody. f: fragments, m: monomer, o: oligomer, *: unspecific bands. Actin was used as loading control. c Intracellular seeding potency of recombinant FL-aSyn and fragments in wild-type (WT) and aSyn knockout (KO) LUHMES cells. Western blots with $\mathrm{N}$ - and C-terminal antibodies in WT cells at DIV6 reveal the same aggregation patterns as in $\mathbf{b}$, whereas no aggregation was detectable in the aSyn knockout cells. In WT cells at DIV10, the aggregation process seems continue after removal of treatment. $\mathrm{f}$ : fragments, m: monomer, o: oligomer, *: unspecific bands. Actin was used as loading control. d Proteinase K (PK) resistance of intracellular aggregates produced by seeding with recombinant FL-aSyn and fragments. PK was added with the indicated concentrations to cell lysates from DIV10 treated cells, and incubated at $37^{\circ} \mathrm{C}$ for $30 \mathrm{~min}$. Intracellular aggregates induced by fragments $61-140$ and 1-95 show a higher PK resistance compared to aggregates formed by recombinant FL-aSyn (1-140) and untreated controls (Ctrl). Actin was used as a control for both sample loading and PK digestion efficiency.

DIV12 (Fig. 6d). To confirm this observation, cells were fixed and stained with 4',6-diamidino-2-phenylindole (DAPI) at DIV12 to quantify the percentage of condensed nuclei representing the final stage of apoptosis (Fig. 6e, f). This method also showed an increased percentage of condensed nuclei in cells treated with fragments 61-140 and 1-95 in comparison to those treated with FL- $\alpha$ Syn and the untreated control.

\section{Discussion}

The specific role of different $\alpha$ Syn fragments in the formation of pathological inclusions remains elusive. Our study demonstrates that $\alpha$ Syn-mediated pathology leads to the appearance of extracellular $\alpha$ Syn fragments. Moreover, recombinant fragments $1-95$ and 61-140 induce very distinct aggregation dynamics of FL- $\alpha$ Syn, result in aggregates with increased PK-resistance, and induce neurotoxicity. Intriguingly, aggregates seeded by these fragments are only recognized by distinct antibodies. Since fragments, in distinction to fibrils, are highly soluble and diffusible, they might be very relevant intercellular spreading species in human diseases and might thus be attractive therapeutic targets.

Isolating extracellularly generated $\alpha$ Syn fragments is technically challenging as their amounts are extremely low. Moreover, since the specific protease(s) responsible for the different fragments detected by LC-MS/MS were 

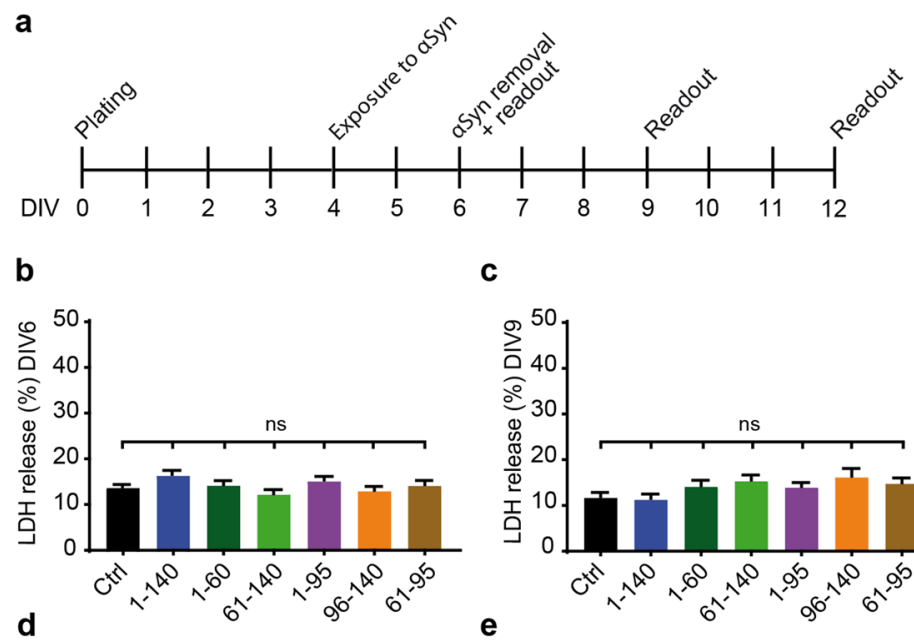

f

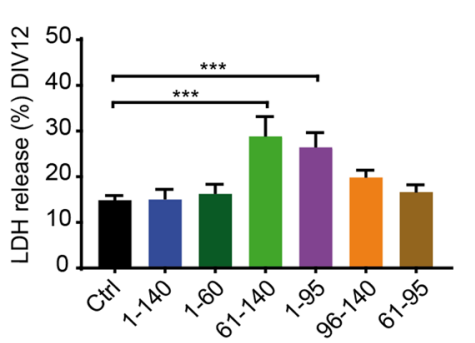

C
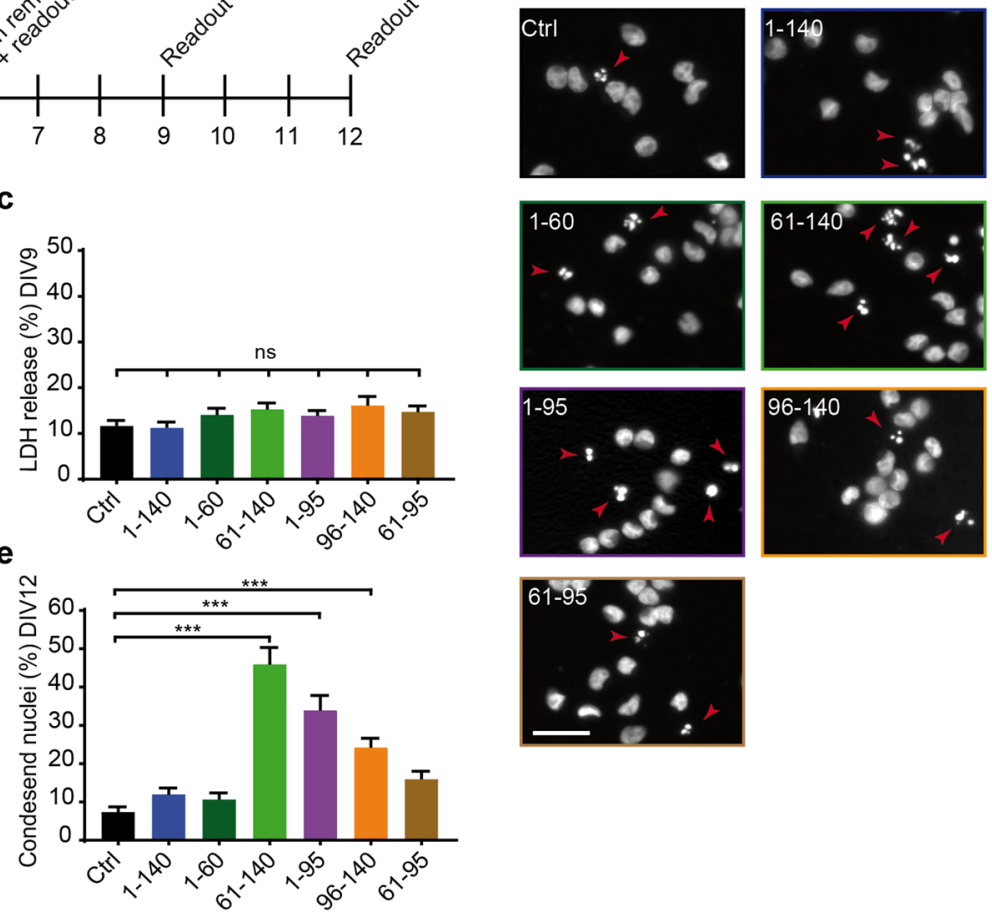

Fig. 6 aSyn fragments that induce intracellular aggregation also induce toxicity. a Experimental design. Cells were differentiated for 4 days before start of treatment. Treatments with recombinant FL-aSyn and fragments were carried out for $48 \mathrm{~h}$. Toxicity was assessed at three readout times. b-d Toxicity measured by LDH release in cells treated with recombinant FL-aSyn and fragments at DIV6 (b), DIV9 (c) and DIV12 (d). At DIV12, recombinant aSyn fragments 61-140 and 1-95 induced toxicity levels of approximately 30\% compared to a cell lysis positive control. Data are presented as mean + SEM from 4 biological repeats with at least 4 technical replicates each. ns: not significant, ${ }^{* * *} p<0.001$; one-way ANOVA with Tukey's post hoc test. e, $\mathbf{f}$ Percentage of condensed nuclei in cells treated with recombinant FL-aSyn and fragments at DIV12. Cells were fixed and nuclei were stained with DAPI. Recombinant aSyn fragments 61-140 and 1-95 induced higher toxicity levels in accordance with LDH data (d). Data are presented as mean + SEM from 3 biological replicates with at least 3 technical repeats each and 3 pictures per technical repeat. ${ }^{* * *} p<0.001$; oneway ANOVA with Tukey's post hoc test. $\mathbf{f}$ Representative pictures of DAPI stained nuclei from cells treated with recombinant FL-aSyn and fragments at DIV12. Red arrowheads indicate condensed nuclei. Scale bar: $30 \mu \mathrm{m}$.

not clearly identified (Fig. 2a), and due to the margin of error inherent to peptide sequencing, the exact sequence of these fragments was not unambiguously ascertained. Thus for further analysis, we used a set of clearly defined recombinant $\alpha$ Syn fragments. Although, the sequences of these recombinant fragments differ from the ones detected by LC-MS/MS, they presented two major advantages. First, they are clearly defined species which are available in required amounts for the study. Secondly, they alternatively combine all the main domains of $\alpha$ Syn, thus allowing the evaluation of the effects of the full domains, rather than the partiallycleaved domains as seen in the fragments detected by LC-MS/MS. Noteworthy, recombinant fragment 1-95 mimics most closely the c-terminally cleaved fragment 11-96 identified in the CM of $\alpha$ Syn-overexpressing cells. This fragment indeed showed striking effects. The other recombinant fragments used in our experiments did not resemble those observed in the supernatant, and may thus be considered as control peptides. Although their sequences may not be considered 'biologically accurate', the evaluation of the effect of distinct domains and domain combinations of $\alpha$ Syn revealed interesting insights in regards to their role in aggregation and spreading.

Several cleaved forms of $\alpha$ Syn were previously found in healthy and diseased human brain samples ${ }^{24}$, as well as in biological fluids such as cerebrospinal fluid, blood ${ }^{25-27}$, and saliva ${ }^{28}$. However, whether their presence in biological fluids and brain samples play a role in spreading of pathology in humans is not clearly established thus far. Notably, fragments with the sequences 1-96 and 65-140, which highly correspond to fragments 1-95 and 61-140 used in our study, were identified in human brains ${ }^{29,30}$. To our knowledge, our findings provide first experimental evidence that these two specific $\alpha$ Syn fragments have distinct seeding properties and may be involved in further propagation of $\alpha$ Syn pathology. 
Interestingly, recombinant fragments 1-95 and 61-140 were found to induce toxicity in primary microglia and increase the release of inflammatory cytokines ${ }^{31}$, further underlining their biological relevance since they might play synergetic pathological roles in both neurons and glial cells.

Our results suggest that fragments are generated by extracellular proteolytic cleavage of $\alpha$ Syn. Several extracellular proteases are known to cleave $\alpha$ Syn, e.g. plas$\min ^{32}$, neurosin ${ }^{33,34}$ and several metalloproteinases ${ }^{35-38}$. Although we were not able to identify the active protease (s) in our experimental system, a comparison between our MS data and previously published cleavage sites of $\alpha$ Syn suggests that plasmin and matrix metalloproteases 3 and 9 could be suitable candidates ${ }^{32,36}$. Further investigation is needed upon the identification of these proteases. Overall, incomplete proteolytic cleavage of extracellular $\alpha$ Syn seems to exacerbate $\alpha$ Syn pathology ${ }^{35,36}$. Here, we provide evidence that $\alpha$ Syn lacking either the $\mathrm{N}$ - or the $\mathrm{C}$ terminus leads to neurotoxicity. Hence, it is necessary to carefully approach the modulation of proteolytic activity as a therapeutic strategy. Regarding our findings, it might be most beneficial to inhibit proteolysis that occurs at the $\mathrm{N}$ - and $\mathrm{C}$-termini and promote more specifically cleavage of the NAC domain of $\alpha$ Syn.

While the aggregation-prone characteristic of Cterminally truncated $\alpha$ Syn is well established ${ }^{39-43}$, little is known about the aggregation activity of $\mathrm{N}$-terminally truncated $\alpha$ Syn. In this study, non-aggregated fragments containing distinct $\alpha$ Syn domains and domain combinations were used to seed the aggregation of FL- $\alpha$ Syn with a molar ratio similar to the one found in $\mathrm{LBs}^{12,44,45}$. We show that non-aggregated, NAC domain containing $\alpha$ Syn fragments can act as powerful seeds. They increase the PKresistance of aggregates, despite of distinct aggregation dynamics. Furthermore, recombinant fragments 1-95 and 61-140 induce very distinct intracellular aggregation patterns, emphasizing that various fragments lead to different aggregates thereby evoking the novel hypothesis of fragments as fate determining factors for aggregation.

Remarkably, endogenous $\alpha$ Syn was essential for aggregation initiation and progression, which was also recently shown in vivo ${ }^{46}$. Importantly, intracellular aggregates derived from seeding with fragments 1-95 and 61-140 also induced a higher cellular toxicity than the ones seeded with FL- $\alpha$ Syn. While it is difficult to conclude with certainty that toxicity derives directly from the different types of intracellular aggregates, the observation that toxicity had a delayed onset and only appeared where PKresistant aggregates accumulate overtime indicates that the two events might be related. Together, these findings suggest that seeding with different fragments can potentially lead to the generation of various $\alpha$ Syn strains. $\alpha$ Syn strains are emerging as a potential explanation for why different brain areas and cell types are affected between various synucleinopathies ${ }^{47}$. Presently, the direct implication of $\alpha$ Syn fragments in the differential formation of different strains has never been adressed. Nonetheless, $\alpha$ Syn fragments were found to have variable solubilities in different synucleinopathies, which directly implicates their seeding activities. Indeed, $\alpha$ Syn fragments seem to be more soluble in multiple system atrophy (MSA) than in Parkinson's disease (PD) and dementia with Lewy bodies (DLB) brain samples ${ }^{48}$. Moreover, a comparison across several studies reveals slight differences in $\alpha$ Syn fragment patterns between PD, DLB, and MSA. In PD, larger fragments $(\sim 12-13 \mathrm{kDa})$ seem to be enriched, whereas smaller fragments $(\sim 6-8 \mathrm{kDa})$ appear to be more abundant in DLB. Detectable but less prominent accumulation of $\alpha$ Syn fragments was also detected in $\mathrm{MSA}^{48,49}$. Accordingly, the question arises whether differential proteolytic activities might be a driving mechanism that can determine the pathogenesis of different synucleinopathies. More recently, fibrils prepared with homogenous $\mathrm{N}$ - or C-terminally truncated $\alpha$ Syn were shown to exhibit distinct prion-like cross-seeding activities in mice ${ }^{50}$. Together with our findings, this strongly implicates the contribution of $\alpha$ Syn fragments in the formation of several strains of aggregates. In relation to our data, further characterization of structural and conformational changes, and the ability to maintain the same characteristics throughout several cycles of aggregation, would provide a deeper understanding of whether $\alpha$ Syn fragments effectively lead to formation of different strains.

Intriguingly, aggregates seeded with fragments 1-95 and 61-140 only showed immunoreactivity toward antibodies that target their corresponding seeding species. Thus, the unrecognized epitopes are either hidden or missing. Accordingly, different conformations of FL$\alpha$ Syn could be formed so that only epitopes of the added seeds are exposed during the aggregation. Alternatively, fragments might trigger the conversion of endogenous FL- $\alpha$ Syn into fragmented forms containing only the epitope of the seed. A similar scenario has been observed with fibrils produced with $\mathrm{C}$-terminally truncated $\alpha$ Syn that activated caspase 1 , which in turn converted endogenous $\mathrm{FL}-\alpha \mathrm{Syn}$ into the same C-terminally truncated form ${ }^{42}$.

Notwithstanding, these findings might have implications for immunotherapeutic approaches, which are currently considered as promising disease-modifying strategies for synucleinopathies ${ }^{51}$. To produce therapeutic antibodies that efficiently target extracellular $\alpha$ Syn and prevents it from further spreading, it is crucial to thoroughly characterize spreading $\alpha$ Syn species. Here, we describe different intracellular aggregates that can be 
completely 'invisible' to certain antibodies. Consequently, a comprehensive screening for a variety of epitopes on several forms of patient-derived $\alpha$ Syn could achieve a better estimation on specific species to target. Moreover, it may be necessary to consider disease-, or even patientspecific mixtures of antibodies.

In summary, we explored the potential of distinct $\alpha$ Syn fragments as spreading species of $\alpha$ Syn pathology across neurons. Our findings provide novel experimental evidence of the direct involvement of various forms of truncated $\alpha$ Syn in differential seeding activities, since they were able to drive aggregation into distinct pathways and induce latent neurotoxicity. These multiple characteristics of $\alpha$ Syn fragments strongly emphasize their relevance as promising therapeutic targets for synucleinopathies.

\section{Methods and materials Cell culture}

Undifferentiated Lund human mesencephalic (LUHMES) cells ${ }^{17}$ were expanded on $50 \mu \mathrm{g} / \mathrm{mL}$ poly-Lornithine (Sigma-Aldrich, St. Louis, MO) coated T75 flasks (EasYFlasks, Nunclon DELTA, VWR, Darmstadt, Germany) in DMEM/F12 (Sigma-Aldrich) supplemented with 1\% N2 supplement (Life Technologies, Carlsbad, $\mathrm{CA}$ ) and $0.04 \mu \mathrm{g} / \mathrm{mL}$ basic fibroblast growth factor (bFGF; PeproTech, Rocky Hill, CT).

For experiments, cells were seeded on either T25 flasks or multi-well-plates (Nunc MicroWell plates, Thermo Fisher Scientific, Waltham, MA) sequentially coated with $50 \mu \mathrm{g} / \mathrm{mL}$ poly-L-ornithine (Sigma-Aldrich) and $5 \mu \mathrm{g} / \mathrm{mL}$ bovine fibronectin (Sigma-Aldrich). To induce differentiation of LUHMES cells into dopaminergic neurons, differentiation medium consisting of DMEM/F12 with 1\% $\mathrm{N} 2$ supplement, $1 \mu \mathrm{g} / \mathrm{mL}$ tetracycline, $0.49 \mu \mathrm{g} / \mathrm{mL}$ dibutyryl cyclic-AMP (Sigma-Aldrich) and $2 \mathrm{ng} / \mathrm{mL}$ glial cellderived neurotrophic factor (GDNF; R\&D Systems, Minneapolis, MN) was used. Cell density was kept at 100,000 cells $/ \mathrm{cm}^{2}$ across all flasks and well plate formats. Cells were kept at all times in standard cell culture conditions at $37^{\circ} \mathrm{C}, 5 \% \mathrm{CO}_{2}$, and water-saturated air. Cells were routinely tested for mycoplasma contamination.

\section{Transduction with adenoviral vectors}

Adenoviral vectors expressing human wild-type alpha synuclein $(\alpha$ Syn) or green fluorescent protein (GFP) under a cytomegalovirus promoter (BioFocus DPI, Leiden, Netherlands) were added to LUHMES cells $48 \mathrm{~h}$ after start of differentiation with a multiplicity of infection (MOI) of 2 , as previously described ${ }^{52,53}$. Untreated controls were supplemented with fresh differentiated medium without adenoviral vectors. After $24 \mathrm{~h}$, the adenoviral vectors were removed and cells were washed three times with PBS (Life Technologies, Carlsbad, CA, USA) to remove virus particles. Cells were supplemented with fresh differentiation medium and kept in culture until indicated readout times.

\section{Treatments with recombinant aSyn}

Cells were treated with $3 \mu \mathrm{M}$ recombinant full-length and fragmented wild-type human $\alpha$ Syn (rPeptide, Watkinsville, GA) after 4 days of differentiation. Except for uptake experiments, treatments were kept on the cells for $48 \mathrm{~h}$. Cells were then washed 3 times with PBS and treated with trypsin-EDTA (Life Technologies) for $45 \mathrm{~s}$ at $37^{\circ} \mathrm{C}$ to remove extracellular $\alpha$ Syn, followed by a wash with DMEM/F12 medium supplemented with $10 \%$ fetal calf serum (FCS; Gibco Life Sciences, Carlsbad, CA), then a final wash in PBS. Cells were either harvested immediately after washing or supplemented with fresh differentiation medium and kept in culture until indicated readout times.

\section{Generation of aSyn knockout LUHMES cell line by CRISPR- Cas9 genome editing}

To target all known splicing variants of the SNCA gene and to exclude the possibility of alternative translation initiation, we targeted SNCA exon 4. The 3' portion of SNCA exon 4 and part of its adjacent intron were substituted with an autonomous puromycin resistance cassette on one allele and introducing a frame shift-inducing indel in the same exon on the second allele. The resistance cassette was designed for genome editing in human cells, harboring a human-codon-optimized pac gene ORF, driven by the human eEF1 $\alpha$ promoter and terminated by bpA, interspaced by unique restriction sites for modular exchange of the elements situated in the pUC57 backbone (purEFlip_VE/pUC57). Small guide RNAs (sgRNAs) were designed using Benchling Biology Software 2016 (Benchling, San Francisco, CA, https://benchling.com) against SNCA exon 4 (5'-AGTAGCCCAGAAGACA GTGG-3') and the adjacent $3^{\prime}$ intron (5'-GGAGCAAGATACTTACTGTG-3'). They were then cloned into the pbs-U6-chimaric_RNA sgRNA expression plasmid. A fragment of approximately $2 \mathrm{~kb}$, harboring the exon, was amplified from LUHMES DNA via PCR and inserted into the pCR-Blunt-II vector (Invitrogen, Carlsbad, CA). The portion between guide RNA-binding positions was consecutively substituted by the puromycin selection cassette, disrupting their target recognition sequences and thus producing the final homologous donor vector. For expression of SpCas9 nuclease, the pCAG-Cas9v2-bpA vector was used. All plasmids were amplified in E. coli DH5- $\alpha$ and isolated using PureLink HiPure plasmid purification kits (Invitrogen, Carlsbad, CA, USA).

LUHMES cells were cultured in standard LUHMES growth medium at $37^{\circ} \mathrm{C} / 5 \% \mathrm{CO}_{2}$. All vessels were precoated with 1\% Geltrex (Gibco Life Sciences) in DMEM/ 
F12 (Sigma-Aldrich, St. Louis, MO, USA) at $37^{\circ} \mathrm{C}$ overnight. At $70 \%$ confluency, cells were washed once with PBS and then detached using Accutase (Sigma-Aldrich) for $15 \mathrm{~min}$ at $37^{\circ} \mathrm{C}$. Detached cells were washed in prewarmed $\left(37^{\circ} \mathrm{C}\right)$ growth medium supplemented with $10 \%$ FCS, pelleted for $5 \mathrm{~min}$ at $270 g$, resuspended in growth medium and counted. Two million cells were then repelleted for $5 \mathrm{~min}$ at $100 \mathrm{~g}$ and resuspended in $100 \mu \mathrm{L}$ nucleofection solution (Amaxa Basic Nucleofector Kit Primary Neurons; Lonza, Basel, Switzerland) supplemented with the appropriate plasmids $\left(10 \mu \mathrm{g}\right.$ for $10^{6}$ cells at a mass ratio of 2:1:1:1 [Cas9: homologous donor: exonic sgRNA: intronic sgRNA]). Cells were then immediately transferred into cuvettes and transfected using program C-013 on a Nucleofector 2b device (Lonza, Basel, Switzerland) according to the manufacturer's protocol $^{18}$. Finally, the cells were allowed to recover in $900 \mu \mathrm{L}$ of prewarmed RPMI medium with 20\% B27 (Gibco Life Sciences) at $37^{\circ} \mathrm{C}$ for $10 \mathrm{~min}$ and added to $5 \mathrm{~mL}$ of prewarmed growth medium in a T25 flask. On the following day, cells were washed once with PBS and grown further in $5 \mathrm{~mL}$ of growth medium. After a first expansion of 2 to 3 days, transfected cells were re-plated in T25 flasks and supplemented with $0.2 \mu \mathrm{g} / \mathrm{mL}$ puromycin on the next day. After another 2 to 3 days, cells were washed with PBS and allowed to recover in growth medium for several days. The cells were then re-plated in $5 \mathrm{~mL}$ growth medium in T25 flasks and the medium supplemented with $0.8 \mu \mathrm{g} / \mathrm{mL}$ puromycin on the next day. After another 2 to 3 days, cells were washed and allowed to recover in growth medium. After one additional passage the cells were then seeded at 300 cells per $1.5 \mathrm{~mL}$ growth medium supplemented with $8 \% \mathrm{~B} 27$ and $10 \mu \mathrm{g} / \mathrm{mL}$ ciprofloxacin in 6-well plates and grown for a week at $37{ }^{\circ} \mathrm{C}, 5 \% \mathrm{CO}_{2}$ and $3 \% \mathrm{O}_{2}$. After clonal expansion, the clones were incubated in $0.02 \%$ EDTA/PBS (Sigma-Aldrich) for $4 \mathrm{~min}$ at $37^{\circ} \mathrm{C}$, PBS was added and individual cell patches were transferred by pipette into $300 \mu \mathrm{L}$ pre-warmed growth medium supplemented with 6\% B27 in 48-well plates. Individual clones were expanded and passaged, using a portion of the cell mass for genotyping. Selection criteria were integration of the resistance cassette into one allele and frame shift-inducing indels in the second allele of the SNCA gene. Absence of $\alpha$-synuclein protein was confirmed by Western blot.

\section{Western blotting}

Cells were harvested in M-PER lysis buffer (Thermo Scientific Pierce Protein Biology, Waltham, MA) supplemented with protease and phosphatase inhibitors cocktail (Roche, Basel, Switzerland). Lysis was carried out by 15 min incubation on ice, followed by one freeze-thaw cycle. Cell lysates were cleared by centrifugation at $13,000 \mathrm{~g}$ for $10 \mathrm{~min}$ at $4{ }^{\circ} \mathrm{C}$. Concentration of the supernatant was determined by BCA protein assay kit (Thermo
Scientific Pierce Protein Biology) according to the manufacturer's instructions.

$\mathrm{CM}$ samples were centrifuged at $2000 \mathrm{~g}$ for $10 \mathrm{~min}$ to remove cell debris. Medium was then concentrated for Western blot analysis with a $3 \mathrm{kDa}$ molecular weight cutoff filter (Vivaspin; Sartorius, Göttingen, Germany). The protein content in the medium was quantified using the $660 \mathrm{~nm}$ Protein Assay Reagent (Thermo Scientific Pierce Protein Biology).

Proteins from cell homogenates were loaded on $4-12 \%$ Bis-Tris precast protein gels (Bio-Rad Laboratories, Hercules, CA) and electrophoresis was carried out with MES running buffer. For CM samples, proteins were loaded on 16.5\% Tris-Tricine precast protein gels (Bio-Rad Laboratories) and electrophoresis carried out with Tris-Tricine running buffer. Proteins were transferred on $0.2 \mu \mathrm{m}$ PVDF membranes (Bio-Rad Laboratories) and immediately fixed with $0.4 \%$ formaldehyde as previously described ${ }^{54}$. Membranes were blocked with a 30\% RotiBlock solution (Carl Roth, Karlsruhe, Germany) or 5\% skimmed milk in Tris-buffered saline (TBS) supplemented with 0.05\% Tween-20 (Sigma-Aldrich) (TBST) and incubated overnight with primary antibodies at $4{ }^{\circ} \mathrm{C}$. Correspondent HRP-coupled secondary antibodies were incubated for $1 \mathrm{~h}$ at room temperature, followed by incubation in Clarity Western ECL Substrate (Bio-Rad Laboratories) for visualization. Images were taken with Odyssey Fc (LICOR Biotechnology, Lincoln, NE) imaging system. The following primary antibodies were used: $\mathrm{C}$-terminal antibody: rabbit anti $\alpha$ Syn (1:500; Cell Signaling Technology, Danvers, MA), N-terminal antibody: rabbit anti $\alpha$ Syn [EP1646Y] (1:500; Abcam, Cambridge, UK), rabbit anti GAPDH (1:3000; Merck Millipore, Billerica, MA), HRP coupled $\beta$-actin antibody (1:2000; Cell Signaling Technology). The following secondary antibody was used: antirabbit IgG (1:5000; Vector Laboratories, Burlingame, CA).

\section{LC/MS-MS analysis}

Gel slices $(2 \mathrm{~mm})$ were excised from a Coomassie brilliant blue stained SDS-polyacrylamide gel at the regions between 5 and $18 \mathrm{kDa}$ (fragments and monomer). Gel slices were further cut into small cubes and transferred into individual vials for subsequent protein cleavage by trypsin and chymotrypsin. First, gel pieces were destained with $50 \mathrm{mM}$ ammonium bicarbonate (Ambic) (SigmaAldrich) in water containing 50\% acetonitrile (ACN) (Sigma-Aldrich). Next, $200 \mathrm{ng}$ of trypsin was added for the first digestion step and the samples were incubated for $12 \mathrm{~h}$ at $37^{\circ} \mathrm{C}$. The supernatant was transferred into a fresh vial and the gel cubes were washed once with $50 \mu \mathrm{L}$ of a $100 \mathrm{mM}$ Ambic solution and the wash was combined with the supernatant from the tryptic cleavage. Subsequently, $300 \mathrm{ng}$ of chymotrypsin were added to the gel cubes and samples were incubated for $4 \mathrm{~h}$ at $30^{\circ} \mathrm{C}$. All samples were 
then acidified using 10\% formic acid (FA) and corresponding supernatants of trypsin and chymotrypsin digestion were combined. Peptides were finally eluted twice with $50 \mu \mathrm{L}$ of $50 \%$ ACN containing $0.1 \%$ FA (SigmaAldrich). All samples were vacuum-dried before desalting on a C18 stage tip column (Thermo Fisher Scientific). Desalted samples were then reconstituted in $0.05 \%$ trifluoroacetic acid (Sigma-Aldrich) and chromatographically separated on an U3000 nano-chromatography system (Thermo Fisher Scientific) which was directly coupled to an LTQ orbitrap mass spectrometer (Thermo Fisher Scientific) for online detection of peptides. Peptides were directly loaded on the RP-C18 nano-column packed into an ESI emitter $(120 \times 0.075 \mathrm{~mm}$, filled with ReproSilPur C18-AQ 2.4 $\mathrm{m}$, Dr. Maisch AG, AmmerbuchEntringen, Germany) and subsequently separated via a linear gradient from $3 \% \mathrm{ACN}$ to $35 \% \mathrm{ACN}$ in $50 \mathrm{~min}$. Peptides eluting from the chromatography column were transferred into the mass spectrometer via nanoelectrospray ionization. The mass spectrometer was run in data-dependent acquisition mode, acquiring one survey scan to detect ionized peptide ions and performing 6 fragment ion scans of selected precursor peptide for sequence determination.

\section{Proteomic data analysis}

All raw data files for excised $\alpha$ Syn gel slices were searched in parallel against a human protein database (uniprot, vs 11/2017) using the MaxQuant/Andromeda search algorithm (vs. 1.6.0.6, www.maxquant.org ${ }^{55}$ ).

For comparison with calculated peptide masses, a mass deviation of $20 \mathrm{ppm}$ was allowed for the first search step, for the main search it was adjusted to $6 \mathrm{ppm}$. For MS/MS data a mass accuracy for $0.6 \mathrm{Da}$ was selected. Protein Nterminal acetylation, methionine oxidation and cysteine carbamidomethylation were enabled as post-translational modifications. All obtained results were filtered for $1 \%$ FDR on the peptide level and $5 \%$ on the protein level. Furthermore, at least two peptide sequences had to be associated with reported protein sequences.

For $\alpha$ Syn, $\mathrm{N}$-terminal and $\mathrm{C}$-terminal regions were not covered by the proteomics data. In order to guarantee correct annotation of the covered areas, identified peptides were loaded into Skyline (vs. 18, University of Seattle, www.skyline.ms ${ }^{56}$ ) and the peptide peak areas were annotated manually. Peptide abundance was compared between all samples.

\section{In vitro cleavage assay}

Recombinant FL- $\alpha$ Syn $(1 \mu \mathrm{g})$ was added to $100 \mu \mathrm{L}$ of various unconcentrated media conditions to monitor proteolytic events. The mixture was incubated at $37^{\circ} \mathrm{C}$ for $24 \mathrm{~h}$. Next, $30 \mu \mathrm{L}$ were taken out of the reaction, mixed with reducing XT sample loading buffer (Bio-Rad
Laboratories) and heated at $95^{\circ} \mathrm{C}$ for $5 \mathrm{~min}$. Samples were loaded on in-house casted 16\% Bis-tris gels prior to Western blotting.

\section{Conditioned medium uptake assay}

Wild-type LUHMES were transduced with $\alpha$ Syn overexpressing AV and the resulting conditioned medium (CM) was harvested at DIV8. The medium was first spun at $2000 \mathrm{~g}$ for $10 \mathrm{~min}$ to remove cell debris. CM was concentrated using $3 \mathrm{kDa}$ molecular weight cut-off filters (Vivaspin; Sartorius, Göttingen, Germany) to achieve the desired concentration factors (2X, 5X and 10X) accordingly. DIV8 $\alpha$ Syn knockout LUHMES cells were treated with different concentrations of the conditioned medium at $37^{\circ} \mathrm{C}$ for $6 \mathrm{~h}$. Cells were afterwards thoroughly washed, harvested and cell lysates were used for Western blotting.

\section{Recombinant protein labeling and uptake assays}

For protein uptake, recombinant proteins were labeled with ATTO-488-NHS ester fluorescent dye (ATTO-TEC, Siegen, Germany) according to the manufacturer's instructions. Briefly, protein concentrations were adjusted to $2 \mathrm{mg} / \mathrm{mL}$ and reconstitution buffer was exchanged from Tris to PBS with dialysis. The $\mathrm{pH}$ of the coupling reactions was adjusted to 8.3 with a $0.2 \mathrm{M}$ sodium bicarbonate solution (Sigma-Aldrich). The fluorescent dye was reconstituted with dimethyl sulfoxide (Sigma-Aldrich) to a final concentration of $5 \mathrm{mg} / \mathrm{mL}$ and added to the proteins with a three to ten folds molar excess. The reaction was carried out at room temperature for $1 \mathrm{~h}$ in the dark. The excess unbound dye was removed with Bio-Spin 6 size exclusion spin columns (Bio-Rad Laboratories). Labeling was verified by running the labeled proteins on a gel followed by visualization with a fluorescent filter. For confocal imaging, cells were plated on 8-well ibidi $\mu$-slides (ibidi, Gräfelfing, Germany). After four days of differentiation, cells were treated with $3 \mu \mathrm{M}$ of FL- $\alpha$ Syn or fragments for $48 \mathrm{~h}$. The treatment was removed and cells were washed as described above. CellTrace Calcein redorange (Thermo Fisher Scientific) was used as a cell filling dye prior to fixing with $4 \%$ paraformaldehyde (SigmaAldrich). 4',6-diamidino-2-phenylindole (DAPI; Invitrogen) was used as a nuclear counterstain. Z-stack images were taken using an inverted laser scanning confocal microscope (Zeiss LSM 880, Carl Zeiss, Oberkochen, Germany) using a 40x oil immersion objective with a digital zoom of 2.5. Orthogonal projections were made using the Fiji software (https://imagej.net/fiji).

For automated uptake kinetics assays, cells were seeded on 48 well-plates and treated with labeled $\alpha$ Syn as described above. At the indicated time points, cells were washed twice with PBS and then treated with a trypan blue solution to quench extracellular green fluorescence as previously described ${ }^{57}$. Intracellular fluorescence in live 
cells at each time point was assessed with a CLARIOstar plate reader (BMG Labtech, Offenburg, Germany) using a well-scanning protocol with a $10 \times 10$ matrix.

\section{LDH assay}

Cell death was quantified by measurement of lactate dehydrogenase (LDH) released into the culture medium at the indicated readout times using the fluorescence based CytotoxONE Membrane Integrity Assay (Promega, Fitchburg, WI) according to the manufacturer's instructions. Fluorescence levels were determined with a fluorescence microplate reader CLARIOstar plate reader (BMG Labtech). Cells lyzed with $1 \%$ triton X (SigmaAldrich) were used as a positive control and were considered as the $100 \%$ cell death reference.

\section{Aggregation assays}

The aggregation reactions were prepared as follows: the different fragments that were used as seeds were mixed with monomeric FL- $\alpha$ Syn with a molar ratio of $1: 10$, respectively, with a final concentration of $20 \mu \mathrm{M}$ in $50 \mathrm{mM}$ tris- $\mathrm{HCl}$ buffer at $\mathrm{pH} 7.0$ and $25 \mu \mathrm{M}$ thioflavine $\mathrm{T}$ (ThT; Sigma-Aldrich). Each sample was run in triplicates in black-walled clear bottom 384 well plates (Thermo Fisher Scientific). The final volume of the reactions was $45 \mu \mathrm{L}$. The plates were sealed and allowed to shake at $700 \mathrm{rpm}$ at $37^{\circ} \mathrm{C}$ for $230 \mathrm{~h}$. Aggregation kinetics were determined by measuring ThT fluorescent signal every hour in relative fluorescence units (RFU). The experiment was repeated at least 3 times.

In order to determine the apparent lag time of the different reactions, each data set was normalized by subtraction of its lowest value and division by its highest value. Apparent lag times were determined as the time at which the curves reached $10 \%$ of the elongation phase $\left(\mathrm{T}_{10 \%}\right)$. Growth rate was calculated as follows: Growth rate (RFU/ hour $)=\left(F_{50 \%}-F_{10 \%}\right) /\left(T_{50 \%}-T_{10 \%}\right)$, where $F_{50 \%}$ and $F_{10 \%}$ are the fluorescence values in RFU when the curves reached 50 and $10 \%$ of the elongation phase, respectively. $T_{50 \%}$ and $T_{10 \%}$ are the time points at which the curves reached 50 and $10 \%$ of the elongation phase, respectively.

\section{Dynamic light scattering measurements}

Particle size distributions and zeta potential measurements were obtained with DLS using a Malvern Zetasizer Nano ZSP (Malver Instruments, Malvern, UK). To obtain an estimation of the hydrodynamic radius $\left(r_{\mathrm{h}}\right)$, the StokesEinstein relation was used:

$$
D=\frac{k_{B} T}{6 \pi \eta r_{h}}
$$

where $D$ is the measured diffusion constant of the particle, $k_{\mathrm{B}}$ the Bolzmann constant, $T$ absolute temperature and the viscosity of the medium. The particle diameter $\left(2 \mathrm{xr}_{\mathrm{h}}\right)$ was calculated from the $\mathrm{Z}$-average size from the cumulants fit, using the software provided by Malvern Instruments.

\section{Proteinase $\mathrm{K}$ digestion}

For recombinant fibrils produced as a 1:10 mixture between fragments and FL- $\alpha$ Syn, $3 \mu \mathrm{g}$ of fibrils were digested at $37^{\circ} \mathrm{C}$ with $0.1 \mu \mathrm{g} / \mathrm{mL}$ of proteinase $\mathrm{K}$ (Thermo Fisher Scientific, Waltham, MA, USA) for the indicated amounts of time. The digestion reaction was stopped by mixing in preheated sample loading buffer followed by immediate incubation at $95^{\circ} \mathrm{C}$ for $5 \mathrm{~min}$. The samples were then loaded on $12 \%$ Bis-Tris criterion gels (Bio-Rad Laboratories). After electrophoresis, the gels were stained with Pierce silver staining kit (Thermo Fisher Scientific) according to the manufacturer's instructions.

For cell lysates, $20 \mu \mathrm{g}$ of total cell lysates were digested at $37^{\circ} \mathrm{C}$ with increasing concentrations of proteinase $\mathrm{K}$ for $30 \mathrm{~min}$. The digestion reaction was stopped by mixing in preheated sample loading buffer followed by immediate incubation at $95^{\circ} \mathrm{C}$ for $5 \mathrm{~min}$. The samples were then loaded on 12\% Bis-Tris criterion gels (Bio-Rad Laboratories) and Western blots were carried out as described above.

\section{Statistical analysis}

Biological repeats were defined as independent experiments and technical repeats were defined as repeats within the same biological repeat. Each experiment includes at least 3 independent repeats. Statistical analysis was performed using GraphPad Prism 7.01 (GraphPad Software, La Jolla, CA). The identify outliers test was performed with GraphPad Prism using the ROUT method $(\mathrm{Q}=1 \%)$ and outliers were excluded from statistical analysis. Data are shown as means with error bars representing the standard error of the mean (SEM). Twoway ANOVA and one-way ANOVA were used followed by Tukey's post hoc test, $p$-values $<0.05$ were considered as statistically significant.

\section{Acknowledgements \\ This work was supported by the Deutsche Forschungsgemeinschaft (DFG, German Research Foundation) under Germany's Excellence Strategy within the framework of the Munich Cluster for Systems Neurology (EXC 2145 SyNergy - ID 390857198), the DFG grant (HO2402/6-2, HO2402/18-1 MSAomics), the NOMIS foundation (FTLD project), and the ParkinsonFonds Deutschland (alpha-Synuclein fragments in PD).}

\section{Author details}

'Department of Translational Neurodegeneration, German Center for Neurodegenerative Diseases (DZNE), 81377 Munich, Germany. ${ }^{2}$ Munich Cluster for Systems Neurology (SyNergy), University of Munich, 81377 Munich, Germany. ${ }^{3}$ Department of Neurology, School of Medicine, Technical University of Munich, 81675 Munich, Germany. ${ }^{4}$ Protein Analysis Unit (ZfP), Biomedical Center (BMC), University of Munich, 82152 Planegg, Germany. ${ }^{5}$ Metabolic Biochemistry, Biomedical Center (BMC), University of Munich, 81733 Munich, Germany. ${ }^{6}$ Center for Neuropathology and Prion Research, University of Munich, 81733 Munich, Germany. ${ }^{7}$ Institute of Developmental Genetics, 
Helmholtz Center Munich, 85764 Munich, Germany. ${ }^{8}$ Department of Neurology, Hannover Medical School, 30625 Hannover, Germany

\section{Conflict of interest}

The authors declare that they have no conflict of interest.

\section{Publisher's note}

Springer Nature remains neutral with regard to jurisdictional claims in published maps and institutional affiliations.

Supplementary Information accompanies this paper at (https://doi.org/ 10.1038/s41419-020-2285-7).

Received: 23 October 2019 Revised: 14 January 2020 Accepted: 16 January 2020

Published online: 03 February 2020

\section{References}

1. Maroteaux, L., Campanelli, J. T. \& Scheller, R. H. Synuclein: a neuron-specific protein localized to the nucleus and presynaptic nerve terminal. J. Neurosci. 8 , 2804-2815 (1988)

2. Withers, G. S., George, J. M., Banker, G. A. \& Clayton, D. F. Delayed localization of synelfin (synuclein, NACP) to presynaptic terminals in cultured rat hippocampal neurons. Brain Res. Dev. Brain Res. 99, 87-94 (1997).

3. Drescher, M. EPR in protein science: intrinsically disordered proteins. Top. Curr. Chem. 321, 91-119 (2012).

4. Breydo, L., Wu, J. W. \& Uversky, V. N. Alpha-synuclein misfolding and Parkinson's disease. Biochim. Biophys. Acta 1822, 261-285 (2012).

5. Burre, J. et al. Alpha-synuclein promotes SNARE-complex assembly in vivo and in vitro. Science 329, 1663-1667 (2010).

6. Kamp, F. et al. Inhibition of mitochondrial fusion by alpha-synuclein is rescued by PINK1, Parkin and DJ-1. EMBO J. 29, 3571-3589 (2010).

7. Nemani, V. M. et al. Increased expression of alpha-synuclein reduces neurotransmitter release by inhibiting synaptic vesicle reclustering after endocytosis. Neuron 65, 66-79 (2010).

8. Goncalves, S. \& Outeiro, T. F. Assessing the subcellular dynamics of alphasynuclein using photoactivation microscopy. Mol. Neurobiol. 47, 1081-1092 (2013).

9. Ma, K. L. et al. The nuclear accumulation of alpha-synuclein is mediated by importin alpha and promotes neurotoxicity by accelerating the cell cycle. Neuropharmacology 82, 132-142 (2014).

10. Braak, H. et al. Staging of brain pathology related to sporadic Parkinson's disease. Neurobiol. Aging 24, 197-211 (2003).

11. Muller, C. M. et al. Staging of sporadic Parkinson disease-related alpha-synuclein pathology: inter- and intra-rater reliability. J. Neuropathol. Exp. Neurol. 64, 623-628 (2005).

12. Beyer, K. \& Ariza, A. alpha-Synuclein posttranslational modification and alternative splicing as a trigger for neurodegeneration. Mol. Neurobiol. 47, 509-524 (2013).

13. Tofaris, G. K. et al. Pathological changes in dopaminergic nerve cells of the substantia nigra and olfactory bulb in mice transgenic for truncated human alpha-synuclein(1-120): implications for Lewy body disorders. J. Neurosci. 26, 3942-3950 (2006).

14. Periquet, M., Fulga, T., Myllykangas, L., Schlossmacher, M. G. \& Feany, M. B. Aggregated alpha-synuclein mediates dopaminergic neurotoxicity in vivo. J. Neurosci. 27, 3338-3346 (2007).

15. Daher, J. P. et al. Conditional transgenic mice expressing C-terminally truncated human alpha-synuclein (alphaSyn119) exhibit reduced striatal dopamine without loss of nigrostriatal pathway dopaminergic neurons. Mol. Neurodegener. 4, 34 (2009).

16. Mazzitelli, S. et al. Amyloid-beta 1-24 C-terminal truncated fragment promotes amyloid-beta 1-42 aggregate formation in the healthy brain. Acta Neuropathol. Commun. 4, 110 (2016).

17. Lotharius, J. et al. Progressive degeneration of human mesencephalic neuron-derived cells triggered by dopamine-dependent oxidative stress is dependent on the mixed-lineage kinase pathway. J. Neurosci. 25, 6329-6342 (2005).
18. Schildknecht, S. et al. Generation of genetically-modified human differentiated cells for toxicological tests and the study of neurodegenerative diseases. ALTEX 30, 427-444 (2013).

19. Höllerhage, M. et al. Trifluoperazine rescues human dopaminergic cells from wild-type alpha-synuclein-induced toxicity. Neurobiol. Aging 35, 1700-1711 (2014).

20. Volpicelli-Daley, L. A. et al. Exogenous alpha-synuclein fibrils induce Lewy body pathology leading to synaptic dysfunction and neuron death. Neuron $\mathbf{7 2}$, 57-71 (2011).

21. Luk, K. C. et al. Pathological alpha-synuclein transmission initiates Parkinson-like neurodegeneration in nontransgenic mice. Science 338, 949-953 (2012).

22. Iwai, A. et al. The precursor protein of non-A beta component of Alzheimer's disease amyloid is a presynaptic protein of the central nervous system. Neuron 14, 467-475 (1995).

23. Bodner, C. R., Dobson, C. M. \& Bax, A. Multiple tight phospholipid-binding modes of alpha-synuclein revealed by solution NMR spectroscopy. J. Mol. Biol. 390, 775-790 (2009).

24. Oueslati, A., Fournier, M. \& Lashuel, H. A. Role of post-translational modifications in modulating the structure, function and toxicity of alpha-synuclein: implications for Parkinson's disease pathogenesis and therapies. Prog. Brain Res. 183, 115-145 (2010).

25. Li, Q. X. et al. Platelet alpha- and gamma-synucleins in Parkinson's disease and normal control subjects. J. Alzheimers Dis. 4, 309-315 (2002).

26. Michell, A. W., Luheshi, L. M. \& Barker, R. A. Skin and platelet alpha-synuclein as peripheral biomarkers of Parkinson's disease. Neurosci. Lett. 381, 294-298 (2005).

27. Hong, Z. et al. DJ-1 and alpha-synuclein in human cerebrospinal fluid as biomarkers of Parkinson's disease. Brain 133, 713-726 (2010).

28. Devic, I. et al. Salivary alpha-synuclein and DJ-1: potential biomarkers for Parkinson's disease. Brain 134, e178 (2011).

29. Li, W. et al. Aggregation promoting C-terminal truncation of alpha-synuclein is a normal cellular process and is enhanced by the familial Parkinson's diseaselinked mutations. Proc. Natl. Acad. Sci. USA 102, 2162-2167 (2005).

30. Kellie, J. F. et al. Quantitative measurement of intact alpha-synuclein proteoforms from post-mortem control and Parkinson's disease brain tissue by intact protein mass spectrometry. Sci. Rep. 4, 5797 (2014).

31. Rabenstein, M. et al. Effect of naturally occurring alpha-synuclein-antibodies on toxic alpha-synuclein-fragments. Neurosci. Lett. 704, 181-188 (2019).

32. Kim, K. S. et al. Proteolytic cleavage of extracellular alpha-synuclein by plasmin: implications for Parkinson disease. J. Biol. Chem. 287, 24862-24872 (2012).

33. Kasai, T. et al. Cleavage of normal and pathological forms of alpha-synuclein by neurosin in vitro. Neurosci. Lett. 436, 52-56 (2008).

34. Tatebe, $H$. et al. Extracellular neurosin degrades alpha-synuclein in cultured cells. Neurosci. Res. 67, 341-346 (2010).

35. Sung, J. Y. et al. Proteolytic cleavage of extracellular secreted \{alpha\}-synuclein via matrix metalloproteinases. J. Biol. Chem. 280, 25216-25224 (2005).

36. Levin, J. et al. Increased alpha-synuclein aggregation following limited cleavage by certain matrix metalloproteinases. Exp. Neurol. 215, 201-208 (2009).

37. Joo, S. H. et al. Regulation of matrix metalloproteinase-9 and tissue plasminogen activator activity by alpha-synuclein in rat primary glial cells. Neurosci. Lett. 469, 352-356 (2010).

38. Oh, S. H. et al. The cleavage effect of mesenchymal stem cell and its derived matrix metalloproteinase-2 on extracellular alpha-synuclein aggregates in Parkinsonian models. Stem Cells Transl. Med. 6, 949-961 (2017).

39. Murray, I. V. et al. Role of alpha-synuclein carboxy-terminus on fibril formation in vitro. Biochemistry 42, 8530-8540 (2003).

40. Ulusoy, A., Febbraro, F., Jensen, P. H., Kirik, D. \& Romero-Ramos, M. Coexpression of C-terminal truncated alpha-synuclein enhances full-length alpha-synuclein-induced pathology. Eur. J. Neurosci. 32, 409-422 (2010).

41. Iyer, A. et al. C-Terminal truncated alpha-synuclein fibrils contain strongly twisted beta-sheets. J. Am. Chem. Soc. 139, 15392-15400 (2017).

42. $\mathrm{Ma}$, L. et al. C-terminal truncation exacerbates the aggregation and cytotoxicity of alpha-Synuclein: A vicious cycle in Parkinson's disease. Biochim. Biophys. Acta Mol. Basis Dis. 1864, 3714-3725 (2018).

43. van der Wateren, I. M., Knowles, T. P. J., Buell, A. K., Dobson, C. M. \& Galvagnion, C. C-terminal truncation of alpha-synuclein promotes amyloid fibril amplification at physiological pH. Chem. Sci. 9, 5506-5516 (2018).

44. Baba, M. et al. Aggregation of alpha-synuclein in Lewy bodies of sporadic Parkinson's disease and dementia with Lewy bodies. Am. J. Pathol. 152 879-884 (1998). 
45. Lucking, C. B. \& Brice, A. Alpha-synuclein and Parkinson's disease. Cell Mol. Life Sci. 57, 1894-1908 (2000).

46. Kim, S. et al. Transneuronal propagation of pathologic alpha-synuclein from the gut to the brain models Parkinson's disease. Neuron 103, 627-641. e627 (2019).

47. Peng, C. et al. Cellular milieu imparts distinct pathological alpha-synuclein strains in alpha-synucleinopathies. Nature 557, 558-563 (2018).

48. Campbell, B. C. et al. The solubility of alpha-synuclein in multiple system atrophy differs from that of dementia with Lewy bodies and Parkinson's disease. J. Neurochem. 76, 87-96 (2001).

49. Liu, C. W. et al. A precipitating role for truncated alpha-synuclein and the proteasome in alpha-synuclein aggregation: implications for pathogenesis of Parkinson disease. J. Biol. Chem. 280, 22670-22678 (2005).

50. Terada, M. et al. The effect of truncation on prion-like properties of alphasynuclein. J. Biol. Chem. 293, 13910-13920 (2018).

51. Brundin, P., Dave, K. D. \& Kordower, J. H. Therapeutic approaches to target alpha-synuclein pathology. Exp. Neurol. 298, 225-235 (2017).
52. Fussi, N. et al. Exosomal secretion of alpha-synuclein as protective mechanism after upstream blockage of macroautophagy. Cell Death Dis. 9, 757 (2018).

53. Höllerhage, M. et al. Multiple molecular pathways stimulating macroautophagy protect from alpha-synuclein-induced toxicity in human neurons. Neuropharmacology 149, 13-26 (2019).

54. Lee, B. R. \& Kamitani, T. Improved immunodetection of endogenous alphasynuclein. PLoS ONE. 6, e23939 (2011).

55. Tyanova, S., Temu, T. \& Cox, J. The MaxQuant computational platform for mass spectrometry-based shotgun proteomics. Nat. Protoc. 11, 2301-2319 (2016).

56. MacLean, B. X. et al. Using skyline to analyze data-containing liquid chromatography, ion mobility spectrometry, and mass spectrometry dimensions. J. Am. Soc. Mass Spectrom. 29, 2182-2188 (2018).

57. Karpowicz, R. J. Jr. et al. Selective imaging of internalized proteopathic alpha-synuclein seeds in primary neurons reveals mechanistic insight into transmission of synucleinopathies. J. Biol. Chem. 292, 13482-13497 (2017) 